\title{
SubTHz Arrays of Planar Antennas with SINIS bolometers for BTA
}

\author{
G. Yakopov ${ }^{4}$, M. Tarasov ${ }^{1}$, A. Gunbina ${ }^{2,5}$, M.Mansfeld ${ }^{2,5}$, R. Yusupov ${ }^{1}$, V. Edelman ${ }^{6}$, \\ and V. Vdovin ${ }^{2,3,5}$ \\ ${ }^{1}$ V.Kotelnikov Institute of Radio Engineering an d Electronics RAS \\ ${ }^{2}$ Institute of Applied Physics RAS \\ ${ }^{3}$ P.N. Lebedev Physical Institute of the Russian Academy of Sciences \\ ${ }^{4}$ Special Astrophysical Observatory RAS \\ ${ }^{5}$ Nizhny Novgorod State Technical University n.a. R.E. Alekseev \\ ${ }^{6}$ P.Kapitza Institute for Physical Problems RAS
}

We have investigated the optical and spectral response of a $350 \mathrm{GHz}$ receiver made of annular antenna array with SINIS bolometer elements. Receiver is intended for using at Big Alt-Azimuthal Telescope (BTA). Samples were measured in a dilution cryostat at temperatures in the range $80-400 \mathrm{mK}$. Voltage responsivity approaches $3 * 10^{9} \mathrm{~V} / \mathrm{W}$. Spectral bandwidth in $230-380 \mathrm{GHz}$ range for single array was measured using Backward Wave Oscillator source.

\section{BTA SAO RAS}

The BTA-6 is a 6-metre aperture optical telescope at the Special Astrophysical Observatory located in the Zelenchuksky District on the north side of the Caucasus Mountains at an altitude of $2070 \mathrm{~m}$ above sea level. Photo and schematic picture of BTA is presented in Fig.1. The mounting of a cryostat with receiving array is supposed in the Nasmyth focus. The equivalent focus length is 184 meters and diameter of diffraction image is $60 \mathrm{~mm}$. For matching receiving array with incoming radiation we can use horn with large aperture (up to $60 \mathrm{~mm}$ ) or lens with the same diameter or decreasing of focal length of 24 times by using a large lens.

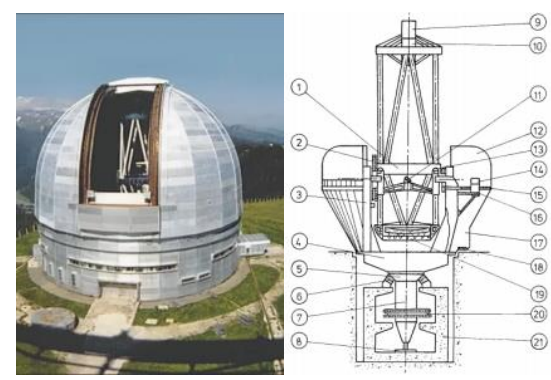

Fig.1. The photo of BTA (left) and schematic picture (right): 1- middle unit; 2 - worm gear; 3 - elevator; 4 - rotation support platform; 5 - spherical support of vertical axis; 6 - spherical pads; 7 - vertical axis; 8 - lower bearing; 9 observer's cage; 10 - prime focus unit; 11 - flat mirror; 12 platform cover; 13 - oil pads; 14 - main spectrograph; 15 observing platform; 16 - support for spectrograph; 17 - pier; 18 - 2m camera mirror of main spectrograph; 19 - primary mirror cell; 20 - spur and worm gears; 21 - reinforced concrete.

\section{Matching of incoming signal with receiver array}

Receiving array can be matched with incoming signal using arrays of horns, quasi-optical lenses or distributed absorbers. The main advantages and dis- advantages of using feedhorns and filled arrays are represented in [1] and in table.

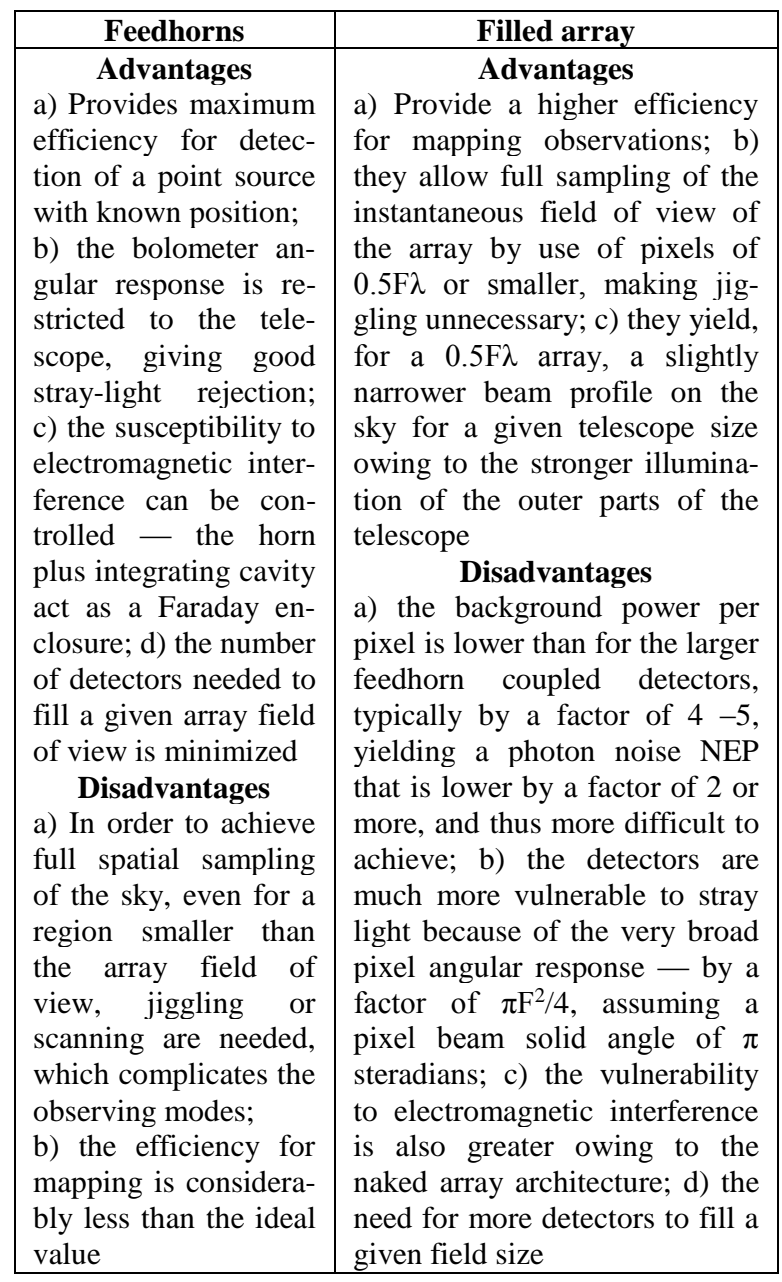

In case of using immersion lens close to elliptical lens [2] allows to remove substrate modes and to increase gain coefficient of planar antenna. The spherical wave transforms in plane wave by mounting the source in the second focus.

We suggest two types of arrays for using at BTA: half-wave antenna arrays (with back-to-back horn) and metamaterial arrays (with lens). A single element is an annular antenna with two (or more) SINIS-bolometers (Fig.2). Schematic image of matching such arrays with incoming radiation are presented in Fig.3.

A metamaterial is made of a periodic array of subwavelength metallic resonators that are collective- 
ly coupled to the free space excitation. In the case of small antennas the matrix can be made more wideband and much smaller that allows placing it in the waist of a single-mode horn or in the focus of immersion lens.

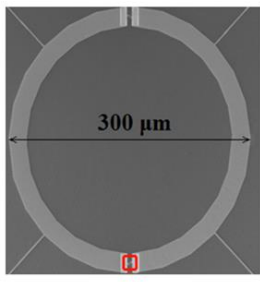

a)

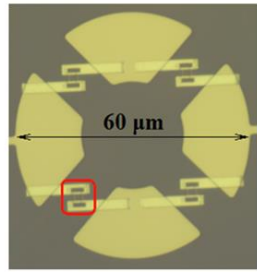

b)

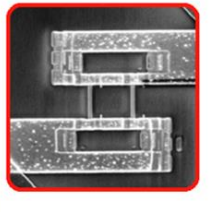

c)
Fig.2. The single elements of investigated structures for $345 \mathrm{GHz}$ receiver arrays: a) Half-wave antenna, b) Metamaterial, c) SINIS-bolometer

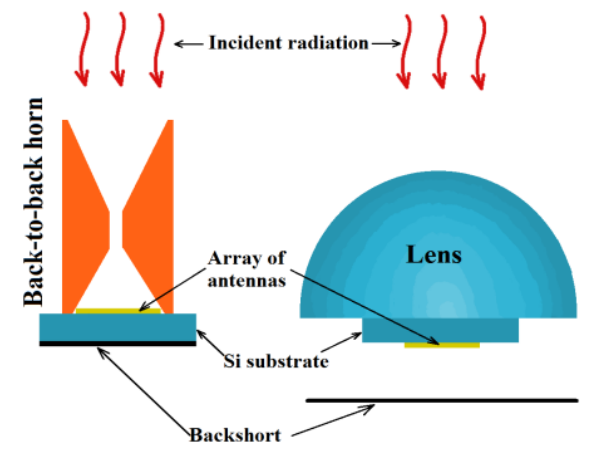

Fig.3. Schematic image of measurements such arrays: halfwave antenna array with back-to-back horn (left) and metamaterial array with lens (right)

\section{Experimental setup and results}

Quasistatic optical response to incoming radiation was measured in dilution cryostat [3] using cold black body (BB) source made of $\mathrm{NiCr}$ film on sapphire substrate. Source was equipped with thermometer, connected to current source and mounted on $2.7 \mathrm{~K}$ stage. For spectral response measurements we use a 230-380 GHz Backward Wave Oscillator (BWO) illuminating the antennas array via optical window and 3 neutral density filters with transmission below $10 \mathrm{~dB}$ placed at radiation shields $100 \mathrm{~K}, 3 \mathrm{~K}, 0.3 \mathrm{~K}$ temperature stages. Two channels were measured simultaneously by lock-in amplifier, one for signal from bolometer, and another from pyroelectric detector that is monitoring level of the incoming power. Schematic pictures of experimental setup are presented in Fig.4. Results of spectral and optical response measurements are presented in Fig.5,6.
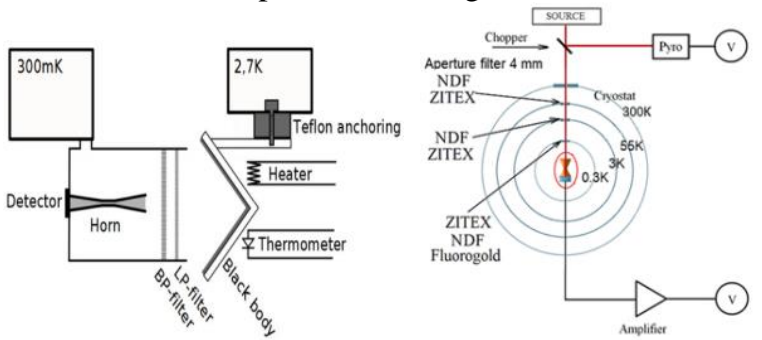

Fig.4. Experimental setup with cold BB (left) and with BWO source (right)

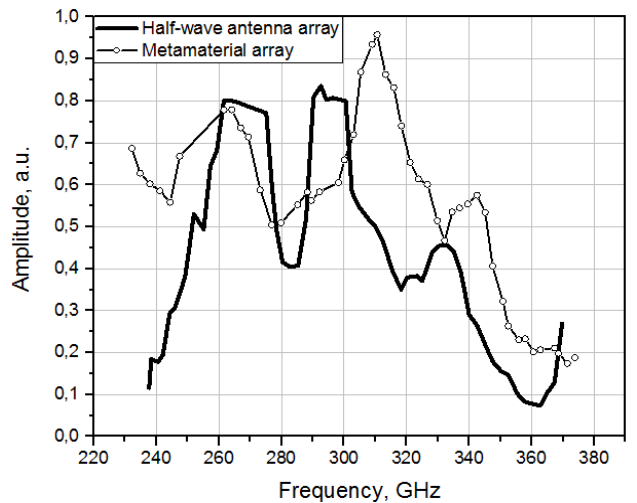

Fig.5. Spectral response of investigated structures

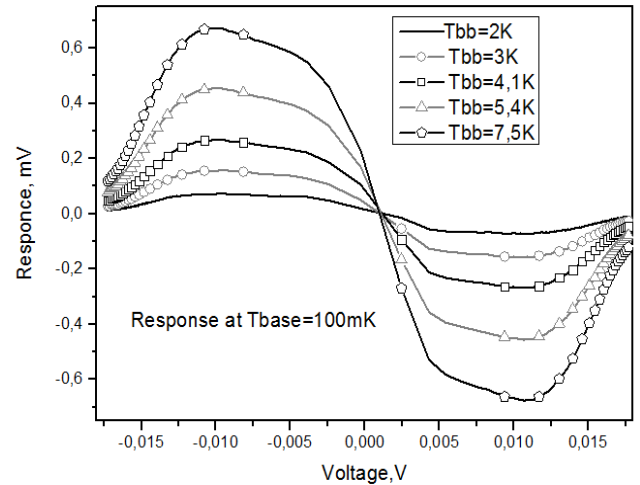

a)

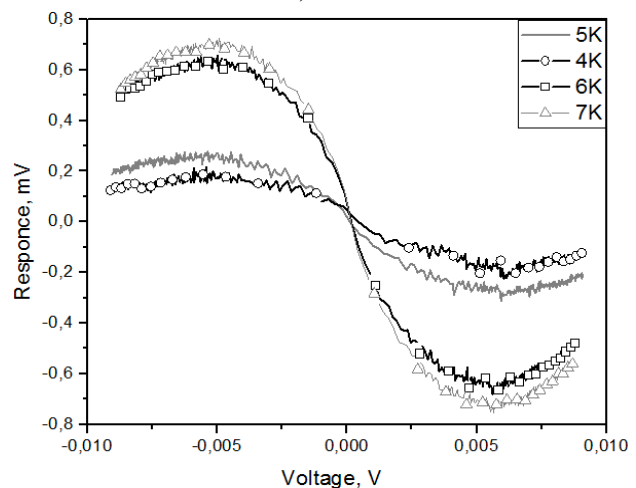

b)

Fig.6. Voltage response of metamaterial array (a) and halfwave antenna array (b) to blackbody radiation

\section{References}

1. M.Griffin, J.Bock, W.Gear, Relative performance of filled and feedhorn-coupled focal-plane architecture, Applied Optics, v/ 41, No31, 6543-6554 (2002)

2. D.F.Filipovic, Double-slot antennas on extended hemispherical and elliptical silicon dielectric lenses, IEEE Trans. Microwave Theor. and Techn., vol. 41, No10, pp.1738-1749 (1993).

3. V. S. Edelman, A dilution microcryostat-insert, Instrum. Exp. Tech. 52, N2, 301-307 (2009), DOI: 10.1134/S002044120902033X.

\section{Acknowledgements}

The work was supported by the IAP RAS state program (project No. 0035-2014-0021) and by the Basic Research Program of the Presidium of the Russian Academy of Sciences No. 32 "Nanostructures: Physics, Chemistry, Biology, Technology Basics". Project "PRIZE" 